\title{
Biomechanical testing of three coracoclavicular ligament reconstruction techniques with a 3D printing navigation template for clavicle-coracoid drilling
}

\author{
$\mathrm{Ji} \mathrm{Qi}^{1,2,3,4 \#} \wedge$, Shijie $\mathrm{Fu}^{5,6,7,8 \#}$, Ruiyue Ping ${ }^{1,3 \# \wedge}$, Kai Wu ${ }^{9 \#}$, Ziyu Feng ${ }^{10}$, Yanxiao $\mathrm{Xu}^{11}$, Xiaoguang Guo ${ }^{5}$, \\ Dingkun Lin ${ }^{1,3}$, Lei Zhang ${ }^{5,6,7,8} \wedge$
}

${ }^{1}$ Department of Orthopaedics, Guangdong Provincial Hospital of Chinese Medicine, Guangzhou, China; ${ }^{2}$ Wang Jing Hospital of China Academy of Chinese Medical Sciences, Beijing, China; ${ }^{3}$ The Second Clinical College of Guangzhou University of Chinese Medicine, Guangzhou, China; ${ }^{4}$ Postdoctoral Research Station, Guangdong Provincial Hospital of Chinese Medicine, Guangzhou, China; ${ }^{5}$ Department of Orthopaedics, Affiliated Traditional Chinese Medicine Hospital of Southwest Medical University, Luzhou, China; ${ }^{6}$ Center for Orthopaedic Diseases Research, Affiliated Traditional Chinese Medicine Hospital of Southwest Medical University, Luzhou, China; ${ }^{7}$ Clinical Base of Affiliated Traditional Chinese Medicine Hospital of Southwest Medical University, Guangdong Province Medical 3D Printing Application Transformation Engineering Technology Research Center, Luzhou, China; ${ }^{8}$ Expert Workstation in Luzhou, Luzhou, China; ${ }^{9}$ The First Affiliated Hospital of Guangzhou University of Chinese Medicine, Guangzhou, China; ${ }^{10}$ Southern Medical University, Guangzhou, China; ${ }^{11}$ Suzhou Yongding Hospital, Suzhou, China

Contributions: (I) Conception and design: J Qi, S Fu, R Ping, K Wu, D Lin, L Zhang; (II) Administrative support: D Lin, L Zhang; (III) Provision of study materials or patients: Z Feng, Y Xu, X Guo; (IV) Collection and assembly of data: J Qi, S Fu, R Ping, K Wu, Y Xu, X Guo; (V) Data analysis and interpretation: Z Feng, X Guo; (VI) Manuscript writing: All authors; (VII) Final approval of manuscript: All authors.

\#These authors contributed equally to this work.

Correspondence to: Dingkun Lin. Professor, Department of Orthopaedics, Guangdong Provincial Hospital of Chinese Medicine, No. 111 Dade Road, Yuexiu District, Guangzhou, China. Email: lindingkun@126.com; Lei Zhang, PhD. Department of Orthopaedics, Affiliated Traditional Chinese Medicine Hospital of Southwest Medical University, No. 182 Chunhui Road, Longmatan District, Luzhou, China. Email: zhanglei870722@126.com.

Background: The identification and precise clavicle-coracoid drilling during coracoclavicular (CC) ligament reconstruction for acromioclavicular (AC) joint dislocation require a high level of experience and surgical skills. Furthermore, the improvement of flexible fixation, such as Endobutton techniques for CC ligament reconstructions is ongoing. We have developed a $3 \mathrm{D}$ printing technique navigation template for clavicle-coracoid drilling and a novel implant for the reconstruction. This study aimed to determine the efficiency of the navigation template for clavicle-coracoid drilling and to evaluate the biomechanical performance of the novel CC ligament reconstruction technique.

Methods: A total of 24 fresh-frozen human cadaveric shoulders were randomly assigned to 1 of 3 reconstruction groups or a control group: TightRope, Triple Endobutton, and the Adjustable ClosedLoop Double Endobutton technique. Computed tomography scans, navigation template designs, and 3D printing were performed for the shoulders. Then, AC joint dislocation was simulated in the reconstruction groups, and $3 \mathrm{CC}$ ligament reconstruction techniques were operated via the 3D printing template separately. Furthermore, biomechanical protocols including the translation test (load from 5 to $70 \mathrm{~N}$ ) and the loadto-failure test were performed to characterize the behaviors and strengths. One-way ANOVA test analyzed differences in displacement under the translation load and the load at failure.

Results: CC ligament reconstructions were performed successfully along with the 3D printing navigation template in the 3 reconstruction groups. During the translation test, no significant difference was found in displacements among the 4 groups. Meanwhile, the mean load of all reconstruction groups at failure (Adjustable Closed-Loop Double Endobutton, 722.1620 N; TightRope, 680.4020 N; Triple Endobutton,

^ ORCID: Ji Qi, 0000-0002-2267-532X; Ruiyue Ping, 0000-0002-9528-9251; Lei Zhang, 0000-0002-7834-4693. 
868.5762 N) was significantly larger than the control group (564.6264 N, P<0.05). The Triple Endobutton group had the maximum load at failure $(\mathrm{P}<0.05)$, however, no significant difference was noticed between the other 2 reconstruction groups $(\mathrm{P}>0.05)$.

Conclusions: The $3 \mathrm{D}$ printing navigation template may become helpful and reliable for AC joint dislocation surgery. Among the $3 \mathrm{CC}$ ligament reconstruction techniques, the Triple Endobutton technique has the best strength in terms of biomechanics, while the biomechanical strength of the Adjustable ClosedLoop Double Endobutton technique is reliable in comparison with the TightRope technique.

Keywords: Acromioclavicular joint dislocation; coracoclavicular ligament reconstruction; 3D printing; Endobutton; biomechanics

Submitted Feb 16, 2021. Accepted for publication Jun 10, 2021.

doi: $10.21037 /$ atm-21-737

View this article at: https://dx.doi.org/10.21037/atm-21-737

\section{Introduction}

Acromioclavicular (AC) joint dislocation is a common injury occurring in the shoulder. In the condition of Rockwood type IV-VI AC joint dislocation, the coracoclavicular (CC) and $\mathrm{AC}$ ligaments are completely torn, and patients may suffer from considerable pain and shoulder dysfunction, requiring reconstruction (1-3). There are over 60 types of techniques for treating AC joint dislocations (4), and implant choice for fixation is one of the most critical management controversies. Rigid fixations in the form of pins, CC screws and hook plate were first developed and are still widely applied in general AC surgeries (5-7), due to their relatively convenient surgical procedures and biomechanical strength. However, such fixations require a second removal procedure, and potential complications may occur, including pain, excessive surgical injuries, osteolysis, and subacromial impingement syndrome (8-11). With the rapid development of sports medicine, clinical evidence has demonstrated that surgical reconstruction by flexible fixation seems to provide a better quality of life for postoperative patients with AC injury (12). Nowadays, it is gradually accepted that the ideal surgical treatment for AC joint dislocation should not only restore its static anatomy, but also flexible fixations (13). Since the CC ligament plays a vital role in the physiological function of the $\mathrm{AC}$ joint, $\mathrm{CC}$ ligament reconstruction has been considered as a practical choice to provide flexible fixation.

The Endobutton technique has become common in CC ligament reconstruction. The primary purpose of this procedure is to achieve flexible fixation with fewer surgical injuries. By applying 2 or 3 titanium-alloy buttons connected by a polyester loop within the distal clavicle and coracoid process, the distance of the CC interval is narrowed, allowing self-restoration of CC ligaments (14). In pursuit of minimal invasiveness and suitable implants, this procedure can also be operated under arthroscopy. Therefore, the technique has gained preference in AC joint repairing surgery $(2,15)$. However, it cannot be ignored that the accuracy of clavicle-coracoid tunnel drilling is of great importance for reconstruction success. To date, it is still one of the difficulties in treating severe injury, especially for inexperienced surgeons. The three-dimensional (3D) printing technique has been popular in surgery (16-19). It may overcome many of the limitations of two-dimensional imaging by generating a graspable $3 \mathrm{D}$ object, and offers better pre-operative planning and accurate navigation during surgery. However, its application in clavicle-coracoid drilling is rare.

Numerous Endobutton techniques such as TightRope (TR) and Triple Endobutton (TE) have been reported in the treatment of AC joint dislocation (20-23). The TE technique exhibited stronger fixation but a more complex surgical process and more significant cost $(22,23)$. The TR technique is easier to perform with less time and cost (20). However, both open-loop design techniques require knotting to achieve the suitable length of the loop, and the reconstructions are prone to failure due to suture breakage. In order to simplify surgical procedures and reduce complications, the closed-loop Endobutton technique has been previously attempted (24).

Meanwhile, the difficulty in adjusting the length of loops has raised more concern. Then, our research team designed another novel modification, namely the Adjustable Closed-Loop Double Endobutton technique (ACLDE). Conveniently, the length of the continuous closed-loop 
could be adjusted by screw rotation above the superior plate without the knotting procedure (25). As one kind of modified technique, the biomechanical performance of ACLDE technique is of vital importance for clinical application in the future. Since the design is similar to the TR and TE techniques and aims to replace the techniques, it is essential to determine whether the ACLDE technique has sufficient biomechanical strength than the other 2 kinds of flexible fixations.

Therefore, this study aimed to design a $3 \mathrm{D}$ printing navigation template to assist accurate clavicle-coracoid drilling during reconstructive surgery. Moreover, via biomechanical tests on cadaveric specimens, the biomechanical performance of ACLDE was evaluated compared to the TR and TE techniques.

We present the following article in accordance with the MDAR reporting checklist (available at https://dx.doi. org/10.21037/atm-21-737).

\section{Methods}

\section{Ethics statement}

All procedures performed in this study involving human participants were following the Declaration of Helsinki (as revised in 2013). The procedures were approved by the Medical Ethics Review Board of Affiliated Traditional Chinese Medicine Hospital of Southwest Medical University (No. KY2018032). Written informed consent was obtained from all donators and preserved by the Department of Anatomy, Southern Medical University.

\section{Specimens and grouping}

This study used 24 fresh-frozen shoulder specimens with native clavicle and scapula from deceased donors. Specimens with obvious deformities or fractures were excluded. A total of 8 female and 16 male donors had a mean age of 43 years (range, 28-60 years). All cadavers were provided by the Department of Anatomy, Southern Medical University, which abides by the local rules of ethics. All specimens were randomly divided into 4 groups: the ACLDE group $(n=6)$, the TR group $(n=6)$, the TE group $(n=6)$, and the control group $(n=6)$.

\section{Design and 3D printing of the navigation template}

Before reconstruction, a thin-slice computed tomography
(CT) scan (Siemens 64-slice spiral CT) was performed for each specimen. The slice thickness was $0.6 \mathrm{~mm}$, in order to ensure the accuracy of $3 \mathrm{D}$ reconstructions. The scan results were saved as data imaging and communications in medicine (DICOM) format files. Then, the navigation template was designed via the MedCAD/cylinder order in Geomagic Studio 2017 (Geomagic, Inc., Cary, North Carolina, USA) and Mimics 21.0 (Materialise, Belgium), following previously reported methods $(18,26,27)$. Firstly, CT data were imported, and the threshold was set to $226-1,688 \mathrm{HU}$. After separation of bone tissue via the thresholding tool, the $3 \mathrm{D}$ models of the AC joint with intact clavicle and scapula were reconstructed via the Calculate $3 \mathrm{D}$ tool. $3 \mathrm{D}$ models were exported as Standard Triangulation Language (STL) format files. Secondly, cutting bone plane was performed on the 3D models to obtain a 5-mm-thick card template fit for the morphology of upper edge of the distal clavicle. Thirdly, the virtual bone tunnels of reconstruction surgery were copied on the models. For the ACLDE and TR groups, one tunnel was drawn from the distal clavicle to the basal part of the coracoid process.

In contrast, for the TE group, the same tunnel and another lateral tunnel were drawn. Each tunnel was set with a $2.75-\mathrm{mm}$ radius column and a $1.5-\mathrm{mm}$ radius inside the channel. Next, Boolean operations (card template + tunnel support column) - (tunnel channel) were implemented to obtain the navigation template (Figure 1).

The final navigation template was output to Makerware via an STL file, and the real-size template was printed by a 3D printing machine (Replicator 2, Makerbot Industries, US). The printer used polylactic acid (PLA), a type of thermoplastic polyester, to extrude the plastic on a build platform where it solidifies. The standard settings were as follows: extruder temperature $215^{\circ} \mathrm{C}$, chamber temperature $24{ }^{\circ} \mathrm{C}$, primary layer height $0.2 \mathrm{~mm}$, infill $2 \%$, support infill $20 \%$, and maximum overhang without support $60 \%$. The printed navigation plate was well-positioned and fixed on the prepared specimen, to assist the clavicle-coracoid drilling (Figure 2).

\section{Specimen preparation and surgical procedures}

Each specimen was thawed at room temperature for 24 hours and free from soft tissue by dissection. The AC joint, CC ligaments, and bony architecture of the scapula and clavicle were left intact. Furthermore, the specimen was confirmed to be free of previous fractures or injuries, and the initial distance of the CC interval was recorded for 

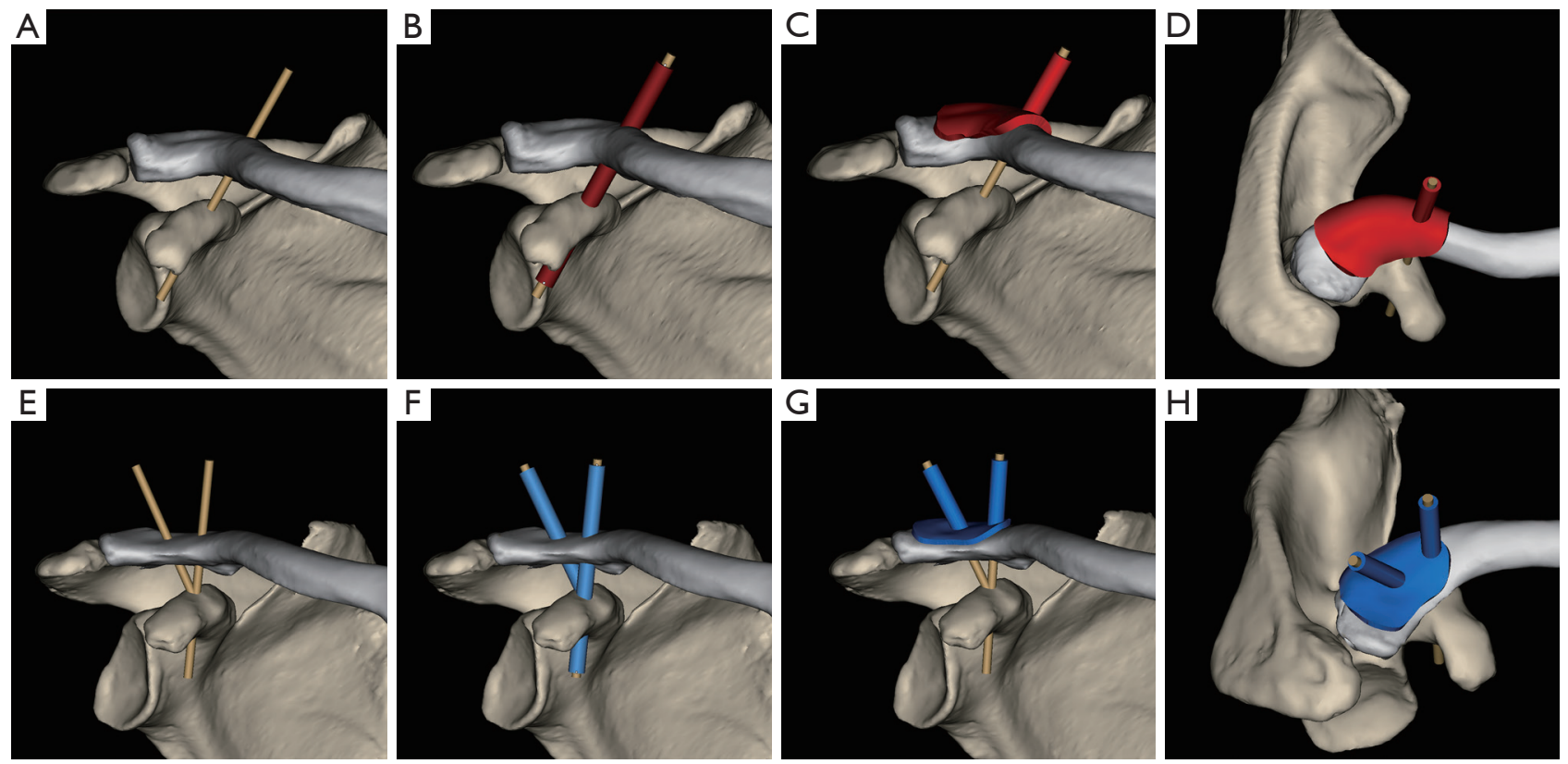

Figure 1 Digital design of the 3D printing navigation template for clavicle-coracoid drilling. (A) A tunnel (1.5 mm in radius) passing through the center of the distal clavicle $(3.5 \mathrm{~cm}$ inside the distal) and the center of the basal part of the coracoid process; (B) column ( $2.75 \mathrm{~mm}$ in radius) connecting the clavicle and coracoid; (C,D) a 5 -mm-thick card template fit for the morphology of the upper edge of the distal clavicle and one-tunnel navigation; (E) double tunnels (1.5 $\mathrm{mm}$ in radius) originating from the center of the distal clavicle (2.5 and 3.5 $\mathrm{cm}$ inside the distal separately) up to the center of the basal part of the coracoid process; (F) double columns (2.75 mm in radius) connecting the clavicle and coracoid; $(\mathrm{G}, \mathrm{H})$ a 5 -mm-thick card template fit for the morphology of the upper edge of the distal clavicle and doubletunnel navigation.

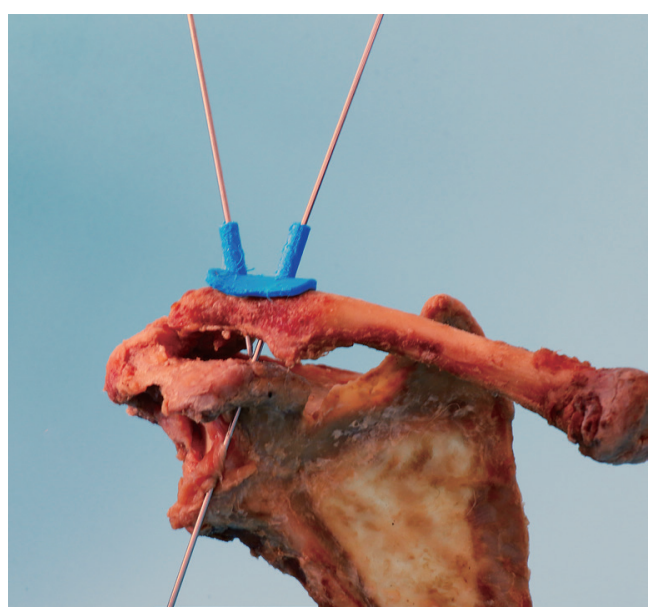

Figure 2 Clavicle-coracoid drilling via the $3 \mathrm{D}$ printing navigation template. reproduction in the subsequent reconstruction. Following previously described methods (28), the AC ligaments and CC ligaments were transected to simulate a complete AC joint dislocation in the ACLDE, TR, and TE groups, and then reconstructed with the 3 techniques separately. The same surgeon performed the procedures.

The ACLDE device consists of one piece of Endobutton titanium-alloy plate, another modified button titaniumalloy plate, and a polyester loop connected together (Delta Medical, Beijing, China; approval by State Intellectual Property Office of the People's Republic of China, CN209122408U). The materials of plates and loop were the same as TR and TE (Figure 3). In the ACLDE group, after the navigation template was well-positioned and fixed, a Kirschner wire (K-wire, $\varphi 1.5 \mathrm{~mm}$ ) was inserted into the 


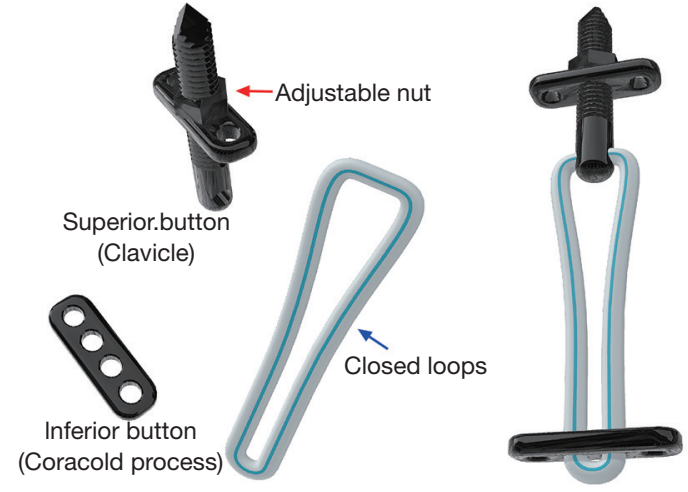

Figure 3 The Adjustable Closed-Loop Double Endobutton (ACLDE) technique. With the closed polyester loop (blue arrow), the titanium-alloy button plates are connected together. Lengthening and shortening of the loop can be performed by nut rotation (red arrow) above the superior plate.

template, drilling from the clavicle to the coracoid process under navigation. Then, the navigation template was pulled out, and the K-wire was fixed as the guide, followed by the second drilling through a hollow drill $(\varphi 4.0 \mathrm{~mm})$. Next, via lead sutures, the implant was inserted into the tunnel, from the clavicle to the coracoid process. After the inferior subcoracoid button was flipped, another plate was left above the clavicle. Finally, the length of the loop was adjusted to fix the joint, by twisting the nut on the superior plate (Figure $4 A, B, C$ ).

In the TR group, after CC drilling like the ACLDE group, the reconstruction by the single-bundle TR system (Arthrex GmbH, Munich, Germany) was performed as previously described (29) (Figure $4 D, E, F$ ). In the TE group, double bone tunnels needed to be drilled in priority under a specific navigation template, and then the TE system (Arthrex GmbH, Munich, Germany) was inserted as previously described $(22,23)$ (Figure 4G,H,I).

\section{Biomechanical protocol}

All biomechanical tests were routinely conducted using the Servo-hydraulic Material Testing System (MTS) and the Win Test Digital Control System (Bose Electro Force 3520-AT; Bose, MN, USA). Throughout all phases of preparation and testing, the specimens were tested at room temperature, and kept moist with $0.9 \%$ saline solution. To evaluate the stability of the primary and reconstructed joints, the translation test was performed in anterior, lateral and superior directions (28). The reference position of the translation test was the acromion adjacent to the AC joint. Firstly, all specimens were conditioned for 10 cycles to $20 \mathrm{~N}$ in anterior, lateral and superior testing to eliminate the creep phenomenon. The translation test (load from $5 \mathrm{~N}$ to $70 \mathrm{~N}$ ) was applied at a rate of $10 \mathrm{~N} / \mathrm{s}$, following the previous method (30). The specimens were then loaded to $70 \mathrm{~N}$ in anterior, lateral, and superior directions, separately (Figure 5).To evaluate the stiffness, the displacements $(5 \mathrm{~N}, 20 \mathrm{~N}, 40 \mathrm{~N}$, $60 \mathrm{~N}$ ) measured by the system were compared among the 4 groups, with an accuracy of $0.0001 \mathrm{~mm}$.

Finally, all specimens were loaded to failure in the superior direction at a constant distraction rate of $1 \mathrm{~mm} / \mathrm{s}$ to assess the maximal tensile loading capacity and the displacement to failure of each group (Figure 5). In addition, no less than a 2-minute interval was maintained before each loading test. Each specimen was preloaded to tighten every laxity in the system and to stretch the construct to a uniform load before measurements began. No specimen was used twice. Thus, each specimen that was reconstructed once was loaded to failure. Failure was defined as rupture of the CC ligament, internal fixation failure, or avulsion fracture (30). Ultimate load at the time of construct failure was record, and the failure mode was visually analyzed. The MTS frame has an accuracy of $0.1 \%$, so the displacement rate of all tests was consistent.

\section{Statistical analysis}

Data were presented as mean and standard deviation (SD). Normal distribution within each group was tested with the Kolmogorov-Smirnov test. For the detection of differences among the 4 groups regarding the displacement under translation load and the ultimate load at failure, one-way ANOVA with Bonferroni post hoc multiple comparisons were used. Analysis was performed by SPSS software (version 20, IBM Corp). A power analysis (a value of 0.05 and power of 0.80 ), based on the estimated load at failure from pilot data and the previous study, showed that a minimum of 6 specimens was required for testing. All statistical tests were two-sided, and the level of statistical significance was set at two-sided $\mathrm{P}<0.05$. 

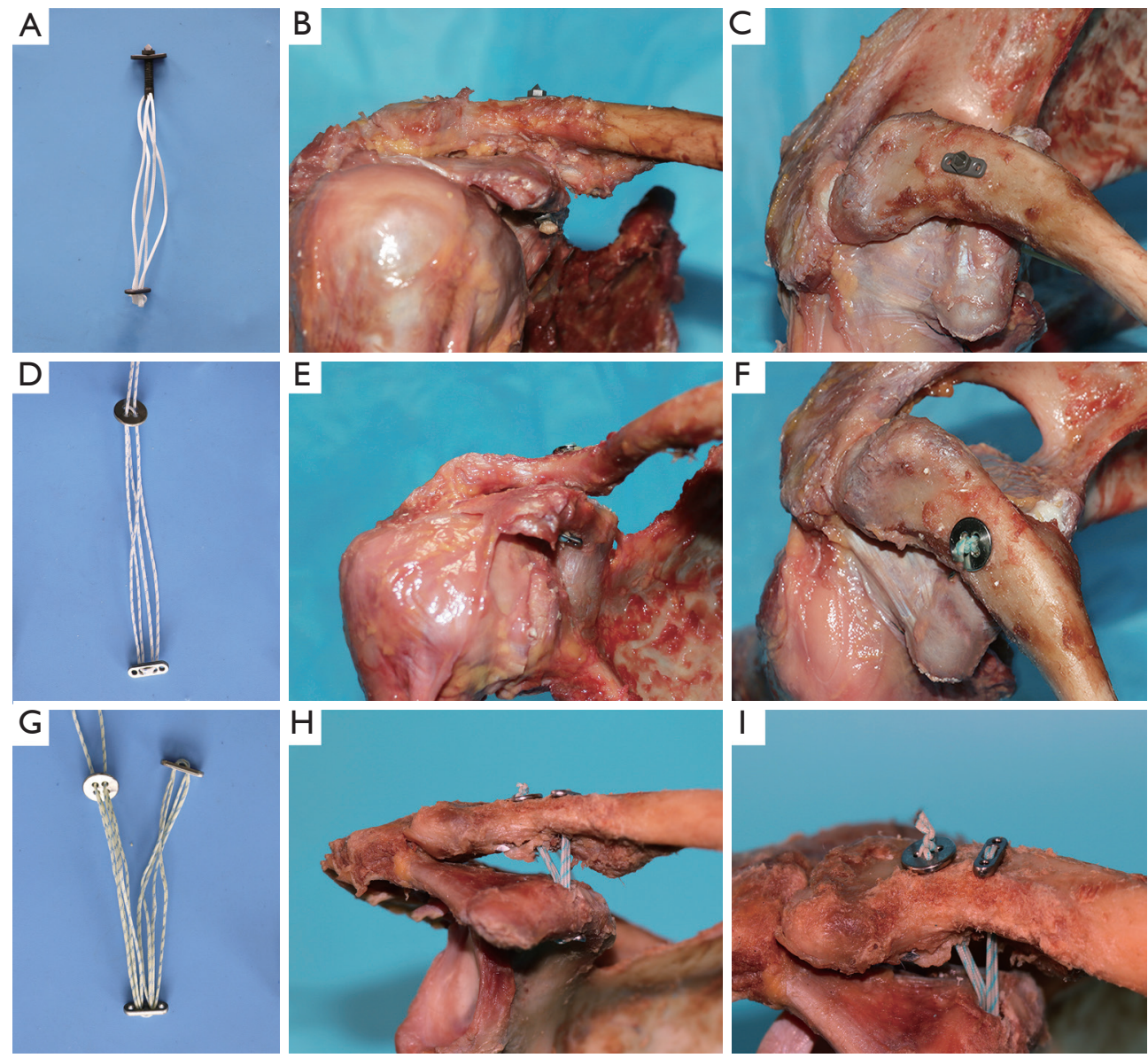

Figure 4 Three coracoclavicular (CC) ligament reconstruction techniques. (A) The Adjustable Closed-Loop Double Endobutton (ACLDE) device; (B) anterior view of the reconstructed acromioclavicular (AC) joint with ACLDE; (C) superior view of the reconstructed AC joint with ACLDE; (D) the TightRope (TR) device; (E) anterior view of the reconstructed AC joint with TR; (F) superior view of the reconstructed AC joint with TR; (G) the Triple Endobutton (TE) device; (H) anterior view of the reconstructed AC joint with TE; (I) superior view of the reconstructed AC joint with TE.

\section{Results}

\section{Clavicle-coracoid drilling}

A total of 24 templates were made via $3 \mathrm{D}$ printing. In 3 reconstruction groups, bone tunnels were drilled successfully through the specially printed templates as previously designed, without any fracture during the drilling procedure.

\section{Translation test}

In the displacements following the anterior, lateral, and superior directions, no significant difference was found among the 4 groups, under the load of 5, 20, 40 and $60 \mathrm{~N}$, respectively $(\mathrm{P}>0.05$; Table 1).

\section{Load-to-failure test}

In the TR group, failure occurred with loop rupture in 2 specimens, clavicle tunnel fracture in 1 specimen, and coracoid process fracture in 3 specimens. In the ACLDE group, failure occurred with loop rupture in 2 specimens, and coracoid process fracture in 4 specimens. In the TE group, failure occurred with coracoid process fracture in 5 specimens, and scapula fracture in 1 specimen.

There was a significant difference in the ultimate load at failure among the 4 groups $(F=13.850, \mathrm{P}=0.00004$; Table 2). The maximum load was found in the TR group $(\mathrm{P}=0.006$ 

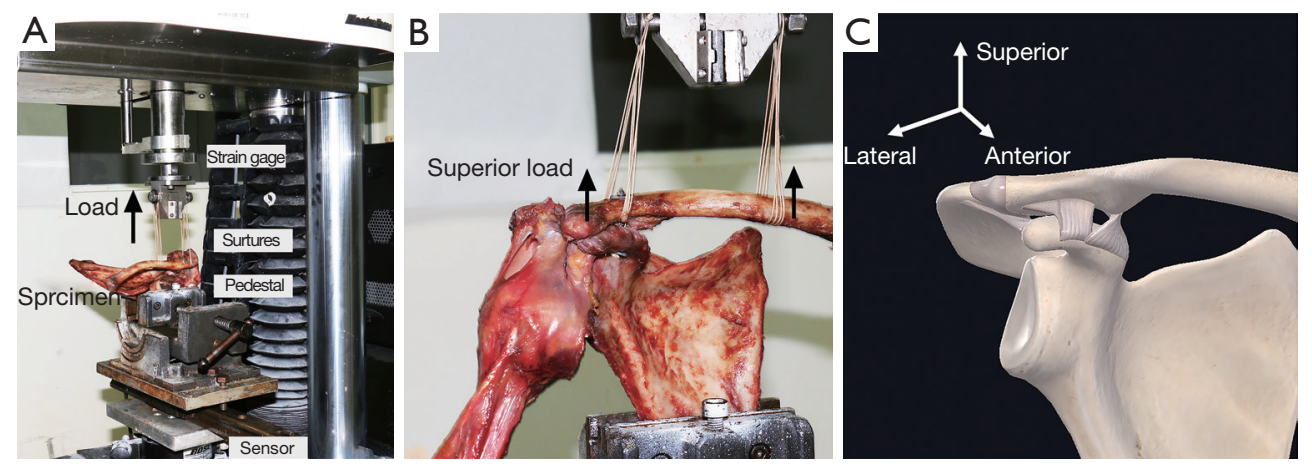

Figure 5 Biomechanical protocols. (A) Tests were conducted using the Bose Electro Force 3520-AT Material Testing System (MTS); (B) load-to-failure: the clavicle was hung by 2 pieces of equidistant ETHIBOND Excel Polyester Sutures (Ethicon, LLC, USA), and the scapular was fixed at the pedestal of MTS at the same time; then, superior load was gradually applied on the specimens until failure; (C) a diagram of the translation test of the acromioclavicular joint in 3 directions (anterior, superior, and lateral), and the load was controlled as no more than $70 \mathrm{~N}$.

Table 1 Difference in anterior, lateral and superior displacement of acromioclavicular joint under translation test among four groups (mean \pm standard deviation)

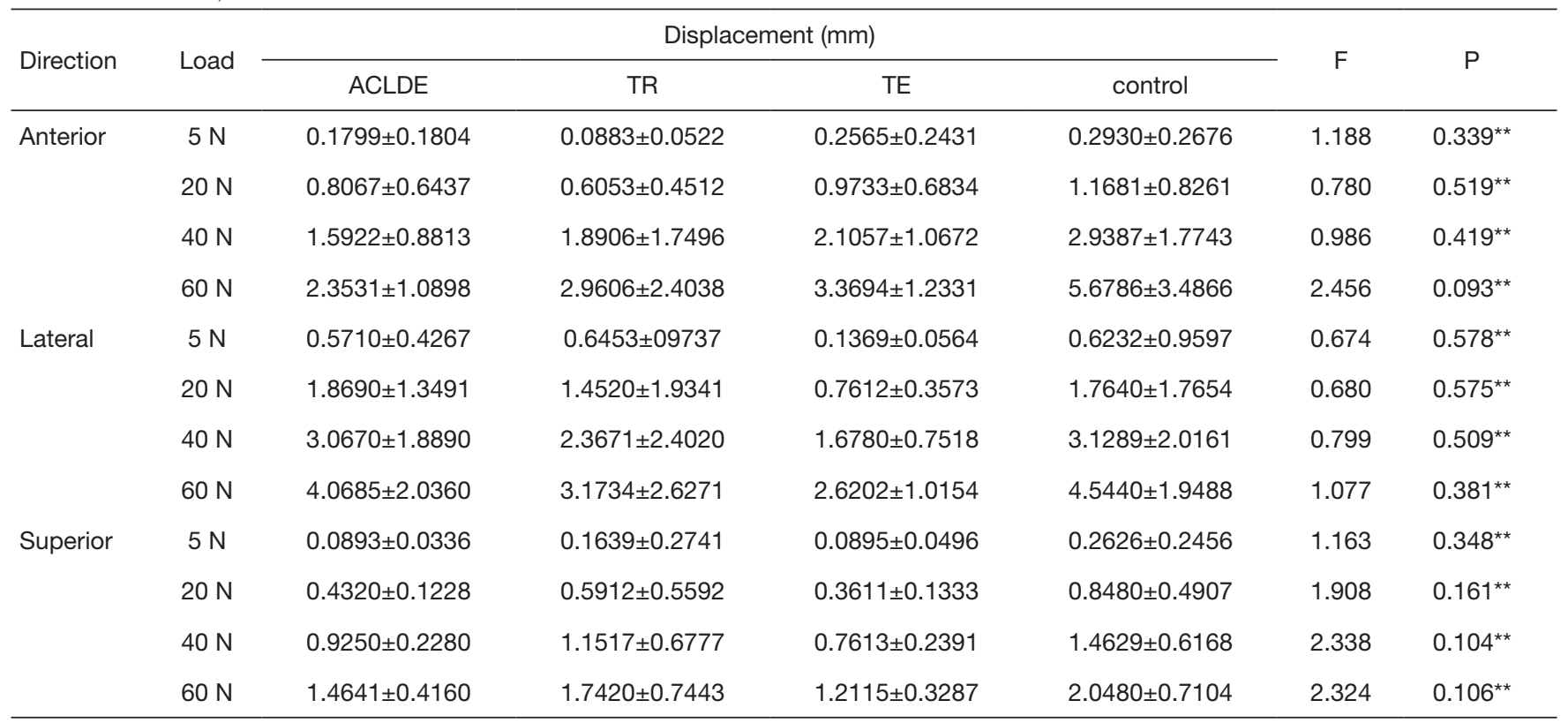

${ }^{* *} \mathrm{P}>0.05$. ACLDE, Adjustable Closed-Loop Double Endobutton; TR, TightRope; TE, Triple Endobutton.

vs. ACLDE group; $\mathrm{P}=0.001$ vs. TR group; $\mathrm{P}=0.000003$ vs. control group), while the minimum load in the control group $(\mathrm{P}=0.004$ vs. ACLDE group; $\mathrm{P}=0.025$ vs. TR group; $\mathrm{P}=0.000003$ vs. TE group). However, no significant difference was observed comparing the ACLDE and TR groups $(\mathrm{P}=0.392)$.

\section{Discussion}

The main advantage of CC reconstruction with Endobutton techniques is achieving flexible fixation, and decreasing the surgical trauma as far as possible. Moreover, the techniques can not only be operated with an open approach, but 
Table 2 Ultimate load $(\mathrm{N})$ at failure of each group

\begin{tabular}{lcccc}
\hline Trial & ACLDE & TR & TE & Control \\
\hline 1 & 824.5644 & 740.2265 & 829.0353 & 543.1532 \\
2 & 665.3798 & 517.5176 & 820.5664 & 566.0421 \\
3 & 834.2998 & 732.9020 & 918.0731 & 456.6088 \\
4 & 733.8176 & 830.8664 & 920.3620 & 568.3310 \\
5 & 609.3020 & 643.1532 & 814.5031 & 627.1554 \\
6 & 665.6086 & 617.7465 & 908.9175 & 626.4678 \\
Mean \pm SD & $722.1620 \pm 92.0401^{\mathrm{a}, \mathrm{c}}$ & $680.4020 \pm 110.2899^{\mathrm{a}, \mathrm{c}}$ & $868.5762 \pm 52.0605^{\mathrm{a}, \mathrm{b}}$ & $564.6264 \pm 63.0537$ \\
\hline${ }^{\mathrm{a}} \mathrm{P}<0.05$ vs. Control group; ${ }^{\mathrm{b}} \mathrm{P}<0.05$ vs. TR group; ${ }^{\mathrm{c}} \mathrm{P}<0.05$ vs. TE group. ACLDE, Adjustable Closed-Loop Double Endobutton; TR, \\
TightRope; TE, Triple Endobutton.
\end{tabular}

also under arthroscopy. Under the limited surgical view, the difficulty of accurate clavicle-coracoid drilling would increase. Also, the fracture of the clavicle or coracoid process along with the inaccurate drilling tunnel may raise concerns. To date, the application of $3 \mathrm{D}$ printing technology in the clinical treatment of AC joint injuries has been rare. Whether it may improve the accuracy of drilling and simply the operation remains unknown. In this study, the special 3D printing navigation template was attempted. Finally, the results revealed that the actual tunnel was accurate as with the former digital design, and the CC ligament reconstructions were performed successfully along with the drilling tunnel in the 3 groups. In addition, the size of the template was suitable for arthroscopic surgery. The important finding indicates that $3 \mathrm{D}$ printing navigation template may be helpful in the future treatment of AC joint dislocations. However, for high-grade AC dislocation, it would be difficult to design the drilling navigation template before surgery. Aiming to solve this problem, we suggested designing the navigation template based on the uninjured side of the anatomical structure.

Of the 3 CC ligament reconstruction techniques used in this study, both the TR and TE techniques have been applied in the clinical treatment of AC dislocation for several years. Such techniques are gaining importance to ensure a lower risk of stiffness, infection, soft tissue morbidity and hardware irritation in postoperative management (31). Numerous biomechanical studies in recent years have found that double-bundle reconstructions such as the TE technique seems to have the advantage of restoring natural biomechanics as far as possible $(4,23)$. Yet, relatively difficult surgical procedures, long operation time, and the higher cost limit more widespread use of the implant. Therefore, TR and other single-bundle reconstructions are well accepted in the clinic. In recent years, numerous biomechanical and clinical studies have accelerated the development of single-bundle reconstructive techniques that are applied to stabilize the AC joint with fewer steps, lower surgical trauma, and more adjustable functions to meet the needs of different patients. The ACLDE device was one of the modified single-bundle devices created by several surgeons. The device's overall design is similar to TR, and the main modification was the adjustable and continuous closed-loop connected with double button plates. When it is fixed on the injured AC joint, the length of the closed-loop can be easily adjusted to meet different needs in a surgical operation via a rotatory screw nut above the superior button plate rather than knotted way. In this way, the implant would increase the convenience of the operation. However, to realize the clinical translation of this technique, biomechanical tests are essential to determine whether the ACLDE system has sufficient biomechanical strength compared with other flexible fixations.

The AC joint has a multidimensional flexible stabilization which is vital to its functioning (32). Considering such biomechanical characteristics, it seemed to be difficult to evaluate the biomechanical behavior of the primary AC joint and reconstructed joint. Previously, tests under the superiorinferior and anterior-posterior dimensional loads were often the focus $(28,29)$, while lateral-medial displacement in the translation test has been rarely investigated. Therefore, this experiment aimed to analyze the displacements under corresponding loads in all 3 dimensions first. During the translation test, to determine the stabilization of internal fixation in the 3 dimensions, the loads were performed on the specimens with no more than $70 \mathrm{~N}$ according to former 
reported methods $(22,30)$. Oki et al. (33) demonstrated that simple abduction of the arm transmitted large axial force, which was up to $34 \mathrm{~N}$ across the AC joint. Previous studies demonstrated that up to $70 \mathrm{~N}$ loading on the specimens was able to simulate the physiological mechanical behavior of the AC joint, and did not affect results during loading in other directions (30). Through the translation test, this study revealed no statistically significant differences in the translation of the AC joint at $70 \mathrm{~N}$ in the superior, anterior, and lateral directions among the ACLDE, TR, TE reconstructions and the intact state. Therefore, it can be demonstrated that the reconstructed AC joint with ACLDE, $\mathrm{TR}$, and TE devices was similar in terms of biomechanical behavior under physiological load. In addition, the 3 types of reconstructions showed similar translation behavior as the control group with an intact AC joint.

Although flexible stabilization of the AC joint was multidimensional, AC joint dislocation occurred mostly in the superior dimension, showing the sticking up distal clavicle on the injured side (30). As a result, this experiment further performed load-to-failure tests in the superior direction, to determine the ultimate strengths of the 3 types of reconstructions and the intact joint. Previously, it has been confirmed that the normal AC joint with native CC ligaments can withstand superior tensile forces up to $500 \mathrm{~N}$ (34). In this study, the results of load-to-failure tests revealed that the mean load at failure of the native AC joint was $564.6264 \mathrm{~N}$, which was in agreement with relevant findings. In contrast, the mean failure load of the AC joint reconstructed by the ACLDE, TR, and TE devices was $722.1620 \mathrm{~N}, 680.4020 \mathrm{~N}$ and $868.5762 \mathrm{~N}$, respectively. These results demonstrated 2 key findings. Firstly, among the 3 reconstructions, the ultimate load of the TE group was significantly higher than the others, indicating that the double-bundle reconstruction would be stronger in ultimate biomechanics than single-bundle reconstruction. In other words, for AC joint dislocation, in comparison with ACLDE and TR devices, CC ligament reconstruction with TE may have lower risk of re-dislocation. Secondly, the study was also aimed at evaluating the ultimate strength of such modified devices as the ACLDE. The results indicated that the superior-inferior biomechanical strength of the ACLDE was better than the normal AC joint. The biomechanical data proved that CC ligament reconstruction with ACLDE technique was reliable in strength.

Moreover, the biomechanical strength was no less than other single-bundle reconstructive devices such as the TR. That is, when single-bundle CC ligament reconstruction for $\mathrm{AC}$ joint dislocation was operated in clinical practice, both the ACLDE and TR techniques were practical choices. Because of the simple operating process of ACLDE without knotting, based on the findings, the study suggests the preferential application of the ACLDE technique. Altogether, the above findings provided basic data of ACLDE devices in biomechanics, suggesting that it would be helpful for clinical application. However, the long-term clinical efficiency and complications remain to be investigated in the future.

Apart from the biomechanical performance, the reasons for the failure of flexible fixations have raised far more concerns. Multiple reasons may induce failure after AC joint reconstruction in clinical practice, such as loop rupture and drill-hole fracture, among others. In biomechanical testing, the failure mode is of high importance for evaluating the performance of a reconstructive technique or device. Thus, it was also recorded in this study. In the ACLDE, TR and TE groups, it was found that the most common mode of failure was a fracture that occurred in the coracoid process, most likely indicating that the relatively vulnerable part of the reconstructions and stress concentration were located on the site of the drilling hole and the inferior sub-coracoid button.

However, there still exist some limitations in this study. Firstly, due to the lack of funding and the limitation of objective conditions, the fresh-frozen shoulder specimens were not all paired, and individual differences might have caused bias in the final results. Secondly, the bone density of relevant cadaveric AC joint structures was undetermined in the study, which leaves room for further improvement of the load-to-failure test. Thirdly, this biomechanical study on cadaveric material could not account for progressive healing of the ligaments. Therefore, it cannot be ignored that the differences among the 4 groups in the study might be less significant in vivo. Fourthly, the study was aimed to determine the strength of the normal and reconstructed joint, but the elasticity was relatively ignored. Lastly, no cycling load was performed to determine the strength of reconstructions, and more biomechanical tests need to be performed in further studies, to characterize more mechanical behaviors of the constructive techniques and implants. Furthermore, the small sample size in this study limits the ability to draw firm conclusions, and these preliminary results will need to be validated in larger studies.

\section{Conclusions}

For the treatment of AC joint dislocation, a 3D printing 
navigation template may be helpful to improve the accuracy of clavicle-coracoid drilling and reduce the difficulty of operations. Moreover, CC ligament reconstruction with the ACLDE, TR, and TE devices have similar biomechanical behaviors as the intact joint under physiological translation load. Among the 3 types of reconstructions, the strength of the TE device was superior to the ACLDE and TR devices in terms of biomechanics. Despite this, the biomechanical strength of the ACLDE device was no less than that of the TR device and the normal joint. Therefore, from a biomechanical perspective, the ACLDE device may become a valid alternative in the clinical treatment of $\mathrm{AC}$ joint dislocation. Furthermore, modification of the technique remains to be performed focusing on the inferior subcoracoid button and bone tunnel site.

\section{Acknowledgments}

Funding: This work was supported by Science Project of Health Commission of Sichuan Province [grant number 20PJ143], Science and Technology Project of Guangdong Province [grant number 2020A1515110966], Science and Technology Project of Guangdong Province [grant number 2020A1414010394], Academician (Shi-zhen Zhong) Talent Team's Subproject of Luzhou Municipal Government and Southwest Medical University [grant number 2018zszysrctdxm1], Strategic Cooperation Project of Luzhou Municipal Government and Southwest Medical University [grant number 2018LZXNYD-ZK43], Science Project of Affiliated Traditional Chinese Medicine Hospital of Southwest Medical University [grant number 2019XYLH-001], and Basic Project of Southwest Medical University and Traditional Chinese Medicine Hospital of Luzhou [grant number 2018-LH003].

\section{Footnote}

Reporting Checklist: The authors have completed the MDAR reporting checklist. Available at https://dx.doi. org/10.21037/atm-21-737

Data Sharing Statement: Available at https://dx.doi. org/10.21037/atm-21-737

Conflicts of Interest: All authors have completed the ICMJE uniform disclosure form (available at https://dx.doi. org/10.21037/atm-21-737). The authors have no conflicts of interest to declare.
Ethical Statement: The authors are accountable for all aspects of the work in ensuring that questions related to the accuracy or integrity of any part of the work are appropriately investigated and resolved. All procedures performed in this study involving human participants were following the Declaration of Helsinki (as revised in 2013). The Medical Ethics Review Board of Affiliated Traditional Chinese Medicine Hospital of Southwest Medical University approved this study (No. KY2018032). Written informed consent was obtained from all donators and preserved by the Department of Anatomy, Southern Medical University.

Open Access Statement: This is an Open Access article distributed in accordance with the Creative Commons Attribution-NonCommercial-NoDerivs 4.0 International License (CC BY-NC-ND 4.0), which permits the noncommercial replication and distribution of the article with the strict proviso that no changes or edits are made and the original work is properly cited (including links to both the formal publication through the relevant DOI and the license). See: https://creativecommons.org/licenses/by-nc-nd/4.0/.

\section{References}

1. Moatshe G, Kruckeberg BM, Chahla J, et al. Acromioclavicular and Coracoclavicular Ligament Reconstruction for Acromioclavicular Joint Instability: A Systematic Review of Clinical and Radiographic Outcomes. Arthroscopy 2018;34:1979-95.e8.

2. Cisneros LN, Reiriz JS. Management of acute unstable acromioclavicular joint injuries. Eur J Orthop Surg Traumatol 2016;26:817-30.

3. Tauber M. Management of acute acromioclavicular joint dislocations: current concepts. Arch Orthop Trauma Surg 2013;133:985-95.

4. Banffy MB, Uquillas C, Neumann JA, et al. Biomechanical Evaluation of a Single- Versus Double-Tunnel Coracoclavicular Ligament Reconstruction With Acromioclavicular Stabilization for Acromioclavicular Joint Injuries. Am J Sports Med 2018;46:1070-6.

5. Eskola A, Vainionpaa S, Korkala O, et al. Acute complete acromioclavicular dislocation. A prospective randomized trial of fixation with smooth or threaded Kirschner wires or cortical screw. Ann Chir Gynaecol 1987;76:323-6.

6. Yoon JP, Lee YS, Song GS, et al. Morphological analysis of acromion and hook plate for the fixation of acromioclavicular joint dislocation. Knee Surg Sports 
Traumatol Arthrosc 2017;25:980-6.

7. McKee MD. Operative Fixation of Chronic Acromioclavicular Joint Dislocation With Hook Plate and Modified Ligament Transfer. J Orthop Trauma 2016;30 Suppl 2:S7-8.

8. Lopiz Y, Checa P, Garcia-Fernandez C, et al. Complications with the clavicle hook plate after fixation of Neer type II clavicle fractures. Int Orthop 2019;43:1701-8.

9. Hoffler CE, Karas SG. Transacromial erosion of a locked subacromial hook plate: case report and review of literature. J Shoulder Elbow Surg 2010;19:e12-5.

10. Chiang CL, Yang SW, Tsai MY, et al. Acromion osteolysis and fracture after hook plate fixation for acromioclavicular joint dislocation: a case report. J Shoulder Elbow Surg 2010;19:e13-5.

11. Leidel BA, Braunstein V, Kirchhoff C, et al. Consistency of long-term outcome of acute Rockwood grade III acromioclavicular joint separations after K-wire transfixation. J Trauma 2009;66:1666-71.

12. Natera-Cisneros L, Sarasquete-Reiriz J, Escola-Benet A, et al. Acute high-grade acromioclavicular joint injuries treatment: Arthroscopic non-rigid coracoclavicular fixation provides better quality of life outcomes than hook plate ORIF. Orthop Traumatol Surg Res 2016;102:31-9.

13. Mori D, Yamashita F, Kizaki K, et al. Anatomic Coracoclavicular Ligament Reconstruction for the Treatment of Acute Acromioclavicular Joint Dislocation. JBJS Open Access 2017;2:e0007.

14. Gowd AK, Liu JN, Cabarcas BC, et al. Current Concepts in the Operative Management of Acromioclavicular Dislocations: A Systematic Review and Meta-analysis of Operative Techniques. Am J Sports Med 2019;47:2745-58.

15. Hashiguchi H, Iwashita S, Abe K, et al. Arthroscopic Coracoclavicular Ligament Reconstruction for Acromioclavicular Joint Dislocation. J Nippon Med Sch 2018;85:166-71.

16. Bachtiar EO, Erol O, Millrod M, et al. 3D printing and characterization of a soft and biostable elastomer with high flexibility and strength for biomedical applications. J Mech Behav Biomed Mater 2020;104:103649.

17. Wu X, Wang G, Rong K, et al. 3D Printed Model Used as Preoperative Tool for Treating Acromioclavicular Joint Dislocation with Pre-Contoured Clavicle Hook Plate: Technical Note. Z Orthop Unfall 2020;158:221-6.

18. Chen X, Zheng F, Zhang G, et al. An experimental study on the safe placement of sacroiliac screws using a $3 \mathrm{D}$ printing navigation module. Ann Transl Med 2020;8:1512.

19. Hoang D, Perrault D, Stevanovic M, et al. Surgical applications of three-dimensional printing: a review of the current literature \& how to get started. Ann Transl Med 2016;4:456.

20. Qi W, Xu Y, Yan Z, et al. The Tight-Rope Technique versus Clavicular Hook Plate for Treatment of Acute Acromioclavicular Joint Dislocation: A Systematic Review and Meta-Analysis. J Invest Surg 2021;34:20-9.

21. Grantham C, Heckmann N, Wang L, et al. A biomechanical assessment of a novel double endobutton technique versus a coracoid cerclage sling for acromioclavicular and coracoclavicular injuries. Knee Surg Sports Traumatol Arthrosc 2016;24:1918-24.

22. Li Q, Hsueh PL, Chen YF. Coracoclavicular ligament reconstruction: a systematic review and a biomechanical study of a triple endobutton technique. Medicine (Baltimore) 2014;93:e193.

23. Wei HF, Chen YF, Zeng BF, et al. Triple endobuttton technique for the treatment of acute complete acromioclavicular joint dislocations: preliminary results. Int Orthop 2011;35:555-9.

24. Zhang L, Zhou X, Qi J, et al. Modified closed-loop double-endobutton technique for repair of rockwood type III acromioclavicular dislocation. Exp Ther Med 2018;15:940-8.

25. Zhang L, He AN, Jin YF, et al. Novel Double Endobutton Technique Combined with Three-Dimensional Printing: A Biomechanical Study of Reconstruction in Acromioclavicular Joint Dislocation. Orthop Surg 2020;12:1511-9.

26. Xu J, He Z, Zhang G, et al. An experimental study on the digital precision of internal fixation via the sinus tarsi approach for calcaneal fractures. J Orthop Surg (Hong Kong) 2019;27:2309499019834072.

27. Yu Z, Zhang G, Chen X, et al. Application of a novel 3D drill template for cervical pedicle screw tunnel design: a cadaveric study. Eur Spine J 2017;26:2348-56.

28. Thomas K, Litsky A, Jones G, et al. Biomechanical comparison of coracoclavicular reconstructive techniques. Am J Sports Med 2011;39:804-10.

29. Lädermann A, Gueorguiev B, Stimec B, et al. Acromioclavicular joint reconstruction: a comparative biomechanical study of three techniques. Journal of Shoulder and Elbow Surgery 2013;22:171-8.

30. Struhl S, Wolfson TS, Kummer F. Axial-Plane Biomechanical Evaluation of 2 Suspensory Cortical Button Fixation Constructs for Acromioclavicular Joint Reconstruction. Orthop J Sports Med 2016;4:2325967116674668. 


\section{Page 12 of 12}

31. Jensen G, Katthagen JC, Alvarado LE, et al. Has the arthroscopically assisted reduction of acute AC joint separations with the double tight-rope technique advantages over the clavicular hook plate fixation? Knee Surg Sports Traumatol Arthrosc 2014;22:422-30.

32. Hislop P, Sakata K, Ackland DC, et al. Acromioclavicular Joint Stabilization: A Biomechanical Study of Bidirectional Stability and Strength. Orthop J Sports Med 2019;7:2325967119836751.

33. Oki S, Matsumura N, Iwamoto $W$, et al. The function of

Cite this article as: Qi J, Fu S, Ping R, Wu K, Feng Z, Xu Y, Guo X, Lin D, Zhang L. Biomechanical testing of three coracoclavicular ligament reconstruction techniques with a $3 \mathrm{D}$ printing navigation template for clavicle-coracoid drilling. Ann Transl Med 2021;9(14):1121. doi: 10.21037/atm-21-737

\section{Qi et al. CC reconstructions with 3D printing navigation template}

the acromioclavicular and coracoclavicular ligaments in shoulder motion: a whole-cadaver study. Am J Sports Med 2012;40:2617-26.

34. Harris RI, Wallace AL, Harper GD, et al. Structural properties of the intact and the reconstructed coracoclavicular ligament complex. Am J Sports Med 2000;28:103-8.

(English Language Editors: C. Betlazar-Maseh and J. Chapnick) 